\title{
Linguistic Validation of the Japanese Version of the Multidimensional Dyspnea Profile and Relation to Physical Activity in Patients with COPD
}

\author{
Masashi Kanezaki (D) \\ Akira Tamaki ${ }^{2}$ \\ Kunihiko Terada ${ }^{3}$ \\ Satoru Ebihara (D) ${ }^{4}$ \\ 'Department of Physical Therapy, School \\ of Health Sciences, Tokyo International \\ University, Kawagoe, Saitama, 350-II97, \\ Japan; ${ }^{2}$ Department of Physical Therapy, \\ School of Rehabilitation, Hyogo \\ University of Health Sciences, Kobe, \\ Hyogo, Japan; ${ }^{3}$ Terada Clinic, Respiratory \\ Medicine and General Practice, Himeji, \\ Hyogo, Japan; ${ }^{4}$ Department of \\ Rehabilitation Medicine, Graduate School \\ of Medicine, Toho University, Tokyo, \\ Japan
}

Correspondence: Masashi Kanezaki Department of Physical Therapy, School of Health Sciences, Tokyo International University, I-I3-I Matobakita, Kawagoe, Saitama, 350-I 197, Japan

Tel +8I-49-232-IIII

Fax +8I-49-232-III

Email mkaneza@tiu.ac.jp
Purpose: This study aimed to develop a linguistically validated Japanese translation of the multidimensional dyspnea profile (MDP) and assess whether worsening of dyspnea's sensory and affective domains during exercise had detrimental effects on physical activity in stable outpatients with chronic obstructive pulmonary disease (COPD).

Materials and Methods: The Japanese version of the MDP was prepared in collaboration with Mapi Research Trust (Lyon, France) after the approval of the developer. Physical activity was assessed using a 3-axis accelerometer. Dyspnea upon exertion was investigated using a 3-minute step test.

Results: The Japanese version of the MDP was obtained and validated linguistically. Airhunger was significantly associated with total calories from walking $(r=-0.47, p<0.05)$, while anxiety and depression were significantly correlated with both the amount and intensity of physical activity $(\mathrm{r}=-0.49, \mathrm{p}<0.05$, and $\mathrm{r}=-0.46, \mathrm{p}<0.05$, respectively).

Conclusion: The Japanese version of the MDP was suggested to reflect both pulmonary functions, ventilatory response during exercise, and intensity and amount of physical activity in patients with COPD.

Keywords: dyspnea, chronic obstructive pulmonary disease, multidimension, physical activity

\section{Introduction}

Dyspnea is a qualitatively distinct subjective sensation of breathing discomfort. ${ }^{1}$ The various sensory qualities of dyspnea have different physiological and emotional consequences, because it is the need for air, but not the sensation of effort while breathing, which induces an unpleasant sensation. ${ }^{2}$ Identifying the sensory and affective components of dyspnea is clinically relevant in managing patients with chronic obstructive pulmonary disease (COPD) who complain of dyspnea during daily physical activity.

Physical inactivity can lead to exacerbation and mortality in patients with COPD $;^{3-6}$ but, many patients limit their physical activity mainly to avoid dyspnea. $^{7}$ There is accumulating evidence that shows physical activity is an important clinical outcome in patients with COPD. ${ }^{8}$ Among the various contributing factors, the effect of breathlessness on physical activity has been a matter of concern. ${ }^{6,7}$ Exertional dyspnea consists of qualitatively distinct sensations that vary in intensity. ${ }^{9,10}$ In particular, a robust inspiratory neural drive upon exertion contributes to the magnitude of dyspnea, ${ }^{11}$ whereas a restricted inspiratory volume 
expansion against the increasing neural respiratory drive and afferent chemoreceptor stimulations lead to air hunger. ${ }^{12}$ Considering that air hunger is more unpleasant than work effort to breathe, affective domains such as anxiety and fear during exercise testing may reflect a subjective affective experience of dyspnea during daily physical activities. ${ }^{10,13}$ Therefore, understanding the sensory and affective elements of dyspnea upon fixed exercise stimulus can play a crucial role in increasing physical activity in patients with COPD.

Banzett et al developed the Multidimensional Dyspnea Profile (MDP), designed to assess immediate breathing discomfort, sensory quality (SQ), and emotional impact of dyspnea during a specific time period. ${ }^{14}$ Because the words used to express breathing sensations may differ, ${ }^{15}$ various versions of the MDP have been widely translated and validated, such as the Danish, ${ }^{16}$ Swedish,,${ }^{17}$ German, ${ }^{18}$ or Portuguese versions. ${ }^{19}$ However, most available instruments in Japanese assess dyspnea only using a monodimensional approach. Therefore, there is a need for an instrument in the Japanese language to evaluate and distinguish multiple aspects of dyspnea at a specific period.

Accordingly, this study aimed to develop a linguistically validated Japanese translation of the MDP and assess whether worsening of dyspnea's sensory and affective domains upon exercise stimulus had detrimental effects on physical activity in stable outpatients with COPD.

\section{Materials and Methods}

\section{Translation}

Structured translation and linguistic validation of the MDP from US English to Japanese was approved by the developer (Robert B. Banzett) and conducted in collaboration with a company specializing in translation and linguistic validation of patient-reported outcome measures (Mapi SAS, Language Services Unit, Lyon, France). In addition, the algorithm of the linguistic validation process was conducted in a structured, multistage process according to International guidelines. ${ }^{20,21}$ It consisted of a forwardtranslation, backward-translation and a cognitive interview step.

After obtaining permission for translation, the original MDP was obtained from the developer. The original was forward-translated into Japanese by two independent professional translators as follows: native American English and Japanese bilingual speakers (Ulatus, Crimson
Interactive Pvt. Ltd, Mumbai, India). The forwardtranslated MDP was analyzed and adjusted by in-country consultants (MK, AT, SE), who provided detailed feedback on the understandability and validity of critical concepts to tool users and people with dyspnea. Forward-translators and in-country consultants discussed all translations and agreed on a reconciled version (version 1) and submitted by MK to the Mapi Research Trust.

To return the first version of the MDP into the source language (version 2), we recruited a native American English and Japanese bilingual speaker (Ulatus, Crimson Interactive Pvt. Ltd, Mumbai, India). The translator for backward translation had no access to the original version. In-country consultants performed a comparison of backward performance with the original MDP. The local coordinator (MK) could contact the backward translator if needed. After submitting the backward translation to Mapi, the developer reviewed it to detect any misunderstandings, mistranslations, or inaccuracies in the first MDP version. Finally, MK submitted a report on the second version of Mapi Research Trust (version 3). Translation version 3 was evaluated through cognitive interviews with five patients with COPD. All participants commented on their understanding of each item. After revising and proofreading, a final linguistically validated translation was issued.

\section{Ethics Approval}

The study was approved by the institutional ethics review board of Himeji Dokkyo University (Himedookusei-1910) and was conducted in accordance with the Declaration of Helsinki. Informed consent was obtained in the form of opt-out by displaying a notice at the clinic.

\section{Study Population: MDP and Physical Activity in COPD}

We contacted 20 patients with stable COPD who had regular outpatient visits at a general practice and respiratory clinic and who agreed to be enrolled in the crosssectional study. Patients with COPD were diagnosed according to the guidelines of the Japanese Respiratory Society, ${ }^{22}$ which was based on a long-term history of smoking, $\mathrm{FEV}_{1} / \mathrm{FVC}<70 \%$ by spirometry after bronchodilator inhalation, and exclusion of other diseases that could cause airflow obstruction. The exclusion criteria were an inability to perform the 3-minute step test, active cardiovascular comorbidity (ie severe heart failure with 
reduced left ventricular ejection fraction, cardiomyopathy, recent acute myocardial infarction, cardiac arrhythmias, or stroke), long-term use of oxygen therapy, or other conditions that could affect dyspnea or exercise capacity.

The patients initially underwent spirometry measurements (CHESTGRAPH HI-301U; Chest Co., Tokyo, Japan) and maximal inspiratory pressure $\left(\mathrm{P}_{\mathrm{I}}, \max \right)$ measurements. $\mathrm{P}_{\mathrm{I}}$, max was measured at functional residual capacity using the POWERbreathe $\mathrm{KH} 2$ device (POWERbreathe International Ltd.). The inspiratory maneuvers were performed with the patient seated and wearing a nose clip. The maneuvers were repeated at least five times until three reproducible efforts (ie $<10 \%$ variance) were obtained. ${ }^{23}$

Appendicular skeletal muscle mass loss was assessed using multifrequency bioelectrical impedance analysis (InBody 470, InBody Japan, Tokyo, Japan). Appendicular skeletal muscle mass was calculated as the sum of the muscle masses of the four limbs, from which the appendicular skeletal muscle mass index was calculated. Quadriceps strength was assessed by the peak force obtained from the isometric maximum knee extension in a sitting position using a belt-fixed hand-held dynamometer ( $\mu$-Tas F-1, Anima, Tokyo, Japan). Handgrip strength was evaluated in a standing position with the elbow straight and the arm held to the body (T.K. K.5401; Takei Kiki Kogyo, Niigata, Japan). ${ }^{9}$ Three measurements were performed, and the highest value was recorded.

\section{Constant-Rate Step Test Protocol}

Constant-exercise testing was performed using the 3-minute step test protocol, consisting of 3-minute bouts of exercise at externally paced stepping rates of 16 steps per minute, as described by Borel et $\mathrm{al}^{24}$ Respiratory flow was measured at rest and during the 3-minute step test through a respiratory flow head (MTL-300L, AD Instruments Ltd, Chalgrove, United Kingdom) attached to a face mask. ${ }^{9}$ Inspiratory capacity maneuvers were performed at rest and the end of the 3-minute step test. ${ }^{9}$ Immediately after each 3-minute step test, the qualitative sensory and affective dimensions of dyspnea were evaluated for each patient using a Japanese MDP questionnaire. ${ }^{14}$ The investigator instructed patients to focus on the end of the 3-minute step test when supplying ratings and selecting feelings. The investigator explained each multidimensional dimensional profile question to every patient. ${ }^{14}$

\section{Physical Activity Measurement}

The Active Style Pro (HJA-750C ${ }^{\circledR}$, Omron Healthcare Inc., Kyoto, Japan) with triaxial acceleration can be worn around the waist and continually records physical activity for two weeks. ${ }^{25}$ This physical activity meter has been validated $^{26}$ and widely used in healthy and elderly people ${ }^{27}$ and patients with COPD. ${ }^{28}$ Each participant was instructed to wear the device for at least 10 hours without sleeping while awake and to avoid water-use activities such as bath time. Ten or more hours of accelerometer wear time per day was considered valid. We measured the number of daily steps and metabolic equivalents (METs) and calculated the total calories consumed due to walking and lifestyle activities.

\section{Statistical Analyses}

Data are presented as means and standard errors. A priori sample size calculation was performed (G*Power3.1.9.2; Universität Kiel) using data from the preliminary investigation and using the previous experimental model in which investigators reported medium to large effect sizes. This indicated that we would need 17 subjects as a total sample size to find statistical significance with an effect size of 0.6 , a power of 0.8 , and an alpha set to 0.05 . Maximal voluntary ventilation (MVV) was calculated from 35 times the $\mathrm{FEV}_{1}$ value. The relationship between MDP score and pulmonary function, ventilatory response, and physical activity was analyzed using Spearman's rank correlation coefficient. $\mathrm{P}$ values $<0.05$ were regarded as statistically significant. Analyses were performed using SPSS (version 27; IBM Corp., Armonk, NY, USA).

\section{Results}

\section{Translation}

A copy of the final certified, linguistically validated Japanese translation of the MDP was developed. The Japanese version of the MDP was produced through forward-translation, backward-translation, and cognitive interviews with patients. It consists of four pages: the first is the A1 scale for measuring discomfort or unpleasantness, the second is the SQ scale for selecting five sensory types, the third measures the intensity of each sensation, and the fourth is the intensity of five emotional items. The MDP item ratings follow a $0-10$ scale: $\mathrm{A} 1: 0=$ neutral; $10=$ unbearable; SQ: $0=$ none, $10=$ as intense as I can imagine; A2: $0=$ none, and $10=$ the highest $I$ can imagine. 
Table I Characteristics of Patients with Chronic Obstructive Pulmonary Disease

\begin{tabular}{|c|c|c|}
\hline Variable & Mean & SD \\
\hline Age (years) & 74.5 & 5.45 \\
\hline Height $(\mathrm{cm})$ & 161.4 & 7.99 \\
\hline Weight (kg) & 57.7 & 11.3 \\
\hline Body mass index $\left(\mathrm{kg} / \mathrm{m}^{2}\right)$ & 22.0 & 3.67 \\
\hline Appendicular skeletal muscle mass $(\mathrm{kg})$ & 22.5 & 4.27 \\
\hline Fat-free mass $(\mathrm{kg})$ & 41.7 & 7.24 \\
\hline $\begin{array}{l}\text { Appendicular skeletal muscle mass } \\
\text { index }\end{array}$ & 6.65 & 1.01 \\
\hline $\mathrm{FEV}_{\mathrm{I}}(\mathrm{L})$ & 1.45 & 0.65 \\
\hline FVC (L) & 2.76 & 0.73 \\
\hline $\mathrm{FEV}_{\mathrm{I}}(\%)$ & 60.2 & 24.50 \\
\hline FVC (\%) & 87.5 & 16.85 \\
\hline IC (L) & 1.99 & 0.51 \\
\hline $\mathrm{P}_{\mathrm{l}, \max }\left(\mathrm{cmH}_{2} \mathrm{O}\right)$ & 69.2 & 30.22 \\
\hline Handgrip strength $(\mathrm{kg})$ & 30.5 & 8.22 \\
\hline Quadriceps strength $(\mathrm{kg})$ & 27.7 & 9.00 \\
\hline
\end{tabular}

Note: Data are expressed as the means and standard deviations.

Abbreviations: $\mathrm{FEV}_{1}$, forced expiratory volume in one second; FVC, forced volume capacity; IC, inspiratory capacity; $\mathrm{P}_{\mathrm{I}}$, max, maximal inspiratory pressure.

\section{Patients}

Twenty patients with COPD were enrolled in this study. The characteristics of pulmonary function, body composition, and muscle strength are shown in Table 1. The participants comprised $80 \%$ males and $74.5 \pm 1.22$ years old, on average. The median body mass index was $22.0 \pm 3.67\left(\mathrm{~kg} / \mathrm{m}^{2}\right)$. Appendicular skeletal muscle mass index and handgrip strength were $6.65 \pm 1.01$ and $30.5 \pm 8.22(\mathrm{~kg})$, respectively.

\section{Relationship Between the Japanese Version of the MDP and Pulmonary Function and Ventilatory Response with Exercise}

Table 2 shows the ventilatory and multidimensional dyspnea profiles in response to the 3-minute step test. As shown in Table 2, dynamic lung hyperinflation was $0.10 \pm 0.29(\mathrm{~L})$ and VE/MVV was $32.8 \pm 30.9$, suggesting that restriction of ventilation during exercise was mild. Regarding the sensory dimension of dyspnea after the 3-minute step test, physical breathing effort was the only variable showing a significant negative
Table 2 Ventilatory and Multidimensional Dyspnea Profile in Patients with Chronic Obstructive Pulmonary Disease

\begin{tabular}{|c|c|c|c|}
\hline & $\begin{array}{c}\text { Ventilatory Response at the End } \\
\text { of the Exercise }\end{array}$ & Mean & SD \\
\hline & $\mathrm{V}_{\mathrm{T}}(\mathrm{L})$ & 0.96 & 0.35 \\
\hline & VE (L) & 23.7 & 8.00 \\
\hline & VE/MVV (\%) & 32.8 & 30.9 \\
\hline & IC at rest $(L)$ & 1.92 & 0.56 \\
\hline & IC at the end of exercise (L) & 1.75 & 0.56 \\
\hline & Change in IC (L) & -0.10 & 0.29 \\
\hline \multicolumn{4}{|c|}{ Multidimensional Dyspnea Profile } \\
\hline \multirow[t]{7}{*}{$\begin{array}{l}\text { Sensory } \\
\text { dimension } \\
\text { SQ scale }\end{array}$} & & & \\
\hline & Physical breathing effort & 1.4 & 1.90 \\
\hline & Air hunger & 2.0 & 2.87 \\
\hline & Tightness & 0.8 & 1.37 \\
\hline & Mental breathing effort & 1.6 & 2.62 \\
\hline & Hyperpnea & 2.2 & 2.80 \\
\hline & Sum of sensory categories & 7.8 & 7.49 \\
\hline \multicolumn{4}{|l|}{$\begin{array}{l}\text { Affective } \\
\text { dimension }\end{array}$} \\
\hline Al scale & Unpleasantness & 4.1 & 2.33 \\
\hline \multirow[t]{6}{*}{ A2 scale } & Depressed & 1.8 & 2.00 \\
\hline & Anxious & 0.4 & 1.05 \\
\hline & Frustrated & 0.2 & 0.62 \\
\hline & Angry & 0.1 & 0.45 \\
\hline & Afraid & 0.5 & 1.28 \\
\hline & Sum of affective categories & 2.9 & 3.85 \\
\hline
\end{tabular}

Note: Data are expressed as the means and standard deviations. Abbreviations: $\mathrm{V}_{\mathrm{T}}$, tidal volume at the end of the exercise; $\mathrm{VE}$, minute ventilation at the end of the exercise; MVV, maximal voluntary ventilation; IC, inspiratory capacity.

correlation with IC immediately after the 3-minute step test $(\mathrm{r}=-0.530, \mathrm{p}<0.05)$. Air hunger after the 3-minute step test was significantly correlated with $\% \mathrm{FEV}_{1}$ and $\mathrm{FEV}_{1} \%$ (Table 3 ). Sense of hyperpnea was significantly associated with inspiratory time and the ratio of inspiratory time to one breath. Regarding the affective dimension of dyspnea after the 3-minute step test, depression was found to be associated with IC, inspiratory muscle strength, and ventilation after the 3-minute step test, and dynamic hyperinflation on exertion; anxiety was associated with ventilation and IC after the 3-minute step test (Table 3).

\section{Relationship Between the Japanese Version of the MDP and Physical Activity}

Table 4 shows the details of the physical activity. Concerning the sensory dimension of dyspnea, the air 
Table 3 Correlation Coefficients Between Multidimensional Dyspnea Profile and Pulmonary Function and Exertional Ventilatory Response in Patients with Chronic Obstructive Pulmonary Disease

\begin{tabular}{|c|c|c|c|c|c|c|c|c|c|c|}
\hline & $\%$ FEV, & $\begin{array}{c}\% \\
\text { FVC }\end{array}$ & FEV $_{1 \%}$ & IC & $\begin{array}{l}P_{\mathbf{l}}, \\
\max \end{array}$ & VE & $t_{1}$ & $t_{1} / t_{\text {tot }}$ & $V_{T} / t_{1}$ & $\begin{array}{c}\text { The Change in } \\
\text { IC }\end{array}$ \\
\hline Physical breathing effort & -0.23 & -0.42 & -0.26 & -0.12 & -0.12 & -0.22 & -0.46 & -0.14 & -0.04 & -0.08 \\
\hline Tightness & -0.13 & -0.08 & -0.18 & 0.07 & -0.08 & 0.21 & -0.33 & 0.06 & 0.07 & 0.04 \\
\hline Air hunger & $-0.60 * *$ & -0.44 & $-0.47^{*}$ & -0.35 & -0.32 & -0.24 & -0.30 & 0.1 & -0.18 & 0.12 \\
\hline Mental breathing effort & -0.19 & -0.04 & -0.24 & -0.06 & -0.13 & -0.16 & -0.47 & -0.07 & 0.12 & 0.09 \\
\hline Hyperpnea & -0.11 & 0.16 & -0.32 & 0.11 & 0.17 & 0.44 & -0.50 & $-0.53 *$ & $0.75 * *$ & -0.07 \\
\hline $\begin{array}{l}\text { Sum of sensory } \\
\text { categories }\end{array}$ & $-0.54 *$ & -0.30 & $-0.58 * *$ & -0.30 & -0.21 & 0.04 & $-0.63 *$ & 0.06 & 0.06 & 0.15 \\
\hline Unpleasantness & -0.19 & 0.15 & -0.17 & -0.37 & -0.40 & 0.12 & $-0.64 *$ & 0.04 & 0.17 & 0.45 \\
\hline Depressed & -0.04 & 0.21 & -0.16 & $0.73 * *$ & $0.57 *$ & $0.47 *$ & 0.4 & -0.42 & 0.32 & $-0.47 *$ \\
\hline Anxious & 0.34 & 0.37 & 0.23 & $0.531 *$ & 0.39 & $0.5 I *$ & 0.07 & -0.38 & 0.46 & -0.06 \\
\hline Frustrated & 0.1 & -0.02 & 0.22 & 0.3 & 0.26 & 0.09 & 0 & -0.31 & 0.12 & -0.40 \\
\hline Angry & -0.09 & -0.15 & -0.03 & 0.35 & 0.28 & 0.22 & -0.14 & -0.14 & 0.09 & -0.44 \\
\hline Afraid & 0.02 & 0.03 & 0 & 0.41 & 0.19 & 0.44 & -0.29 & -0.18 & 0.33 & -0.23 \\
\hline $\begin{array}{l}\text { Sum of affective } \\
\text { categories }\end{array}$ & 0.01 & 0.2 & -0.11 & $0.76 * *$ & $0.57^{*}$ & $0.58 * *$ & 0.18 & -0.40 & 0.41 & -0.45 \\
\hline
\end{tabular}

Notes: Data represent the Spearman correlation coefficient. *And the bold font denote $\mathrm{p}$-value less than 0.05 . **And the bold font denote $\mathrm{p}$-value less than $0.0 \mathrm{I}$.

Abbreviations: $V_{T}$, tidal volume at the end of the exercise; $V E$, minute ventilation at the end of the exercise; IC, inspiratory capacity; $t_{\mathrm{l}}$, inspiratory time; $t_{\mathrm{l}} / t_{\text {tot }}$, duty cycle; $V_{\mathrm{T}} / t_{\mathrm{t}}$, inspiratory flow rate.

hunger after the 3-minute step test showed a significant negative correlation with the calories consumed during walking (Table 5). In contrast, with respect to the affective aspect of dyspnea, depression was found to be significantly correlated with total calories and physical activity duration at intensities $\geq 5$ MET (Table 5). Anxiety in the 3-minute step test was found to be significantly negatively correlated with the total calories consumed during walking (Table 5).

\section{Discussion}

To the best of our knowledge, the Japanese version of the MDP is the first instrument to examine the sensory and affective dimensions of dyspnea during a specific period determined by the user. Dyspnea is a distressing symptom in patients with COPD. Identifying the intensity and SQ of dyspnea is clinically relevant because the physiological and emotional consequences of various dyspnea types differ. $^{2}$ Therefore, a more precise characterization of the sensory and affective dimensions of dyspnea using the
Japanese version of the MDP could help develop better approaches to control dyspnea in a clinical setting.

This study examined only the Japanese linguistic validity of the MDP. To examine whether the individual question items included in the Japanese version of the MDP have internal consistency, Cronbach's alpha reliability coefficients may be determined. However, the magnitude of dyspnea during inspiratory resistive breathing has been reported to decrease in males and increase in females with each breath. ${ }^{29}$ Considering that the MDP assesses sensory and emotional aspects of dyspnea at a specific period, it is difficult to determine the difference between the first experimentally induced and repeated dyspnea in the same participant, given the nature of the modifying effects of dyspnea as described above.

Although physical activity is influenced by multidimensional elements, breathlessness is one of the major limiting factors. ${ }^{7}$ The present study examined the effects on physical activity of the sensory and emotional aspects of dyspnea after an identical exercise load using the Japanese version of the MDP. It was found that the sensation of air hunger was negatively associated with the 
Table 4 Characteristics of Physical Activity in Patients with Chronic Obstructive Pulmonary Disease

\begin{tabular}{|l|c|c|}
\hline Intensity of Physical Activity & Mean & SD \\
\hline$>$ I MET (min/day) & 610.4 & 219.0 \\
\hline$>2$ MET (min/day) & 193.1 & 96.8 \\
\hline$>3$ MET (min/day) & 54.1 & 34.0 \\
\hline$>4$ MET (min/day) & 13.4 & 12.0 \\
\hline$>5$ MET (min/day) & 2.7 & 2.5 \\
\hline Amount of physical activity & & \\
\hline Total calories from walking (kcal/day) & 100.4 & 58.0 \\
\hline Total calories from lifestyle activities (kcal/day) & 355.1 & 148.8 \\
\hline The number of daily steps (step/day) & 3866.6 & 3370.1 \\
\hline
\end{tabular}

Note: Data are expressed as the means and standard deviations.

Abbreviation: MET, metabolic equivalents.

amount of energy spent walking (calories), indicating that air hunger contributed to the avoidance of physical activity. Furthermore, the effect of the emotional aspects of dyspnea on both the amount and intensity of physical activity suggests the need for intervention in these aspects.
The emotional aspect of breathlessness after the 3-minute step test was mild; but, anxiety and depression after the 3-minute step test significantly correlated with physical activity. The scores on the emotional aspect of dyspnea during exercise in patients with COPD are known to be relatively mild. ${ }^{9,30}$ However, despite the mild intensity of the emotional aspect of dyspnea, a recent study has shown that the exertional affective dimension of breathlessness is associated with COPD exacerbation. ${ }^{10}$ This suggests that the emotional aspects of breathlessness upon physical activity and exercise loading need to be carefully evaluated to prevent exacerbations.

Air hunger was associated with $\mathrm{FEV}_{1}(\%)$ but not with dynamic lung hyperinflation. Air hunger can be caused by an inadequate ventilatory response to respiratory drive; the VE/MVV at the end of the 3-minute Step Test was $32.8 \pm$ $7.49 \%$, suggesting that the inadequate ventilatory response to respiratory drive was mild. The change in IC before and after exercise was also modest, suggesting that it was linked to mild air hunger. However, regardless of the modest air hunger upon exertion, the association between air hunger

Table 5 Correlation Coefficients Between Multidimensional Dyspnea Profile and Physical Activity in Patients with Chronic Obstructive Pulmonary Disease

\begin{tabular}{|c|c|c|c|c|c|c|c|c|c|c|}
\hline & $\begin{array}{c}>I \\
\text { MET } \\
(\min )\end{array}$ & $\begin{array}{c}>2 \\
\text { MET } \\
(\mathrm{min})\end{array}$ & $\begin{array}{c}>3 \\
\text { MET } \\
(\min )\end{array}$ & $\begin{array}{c}>4 \\
\text { MET } \\
(\min )\end{array}$ & $\begin{array}{c}>5 \\
\text { MET } \\
(\mathrm{min})\end{array}$ & $\begin{array}{c}>6 \\
\text { MET } \\
(\mathrm{min})\end{array}$ & $\begin{array}{c}>7 \\
\text { MET } \\
(\min )\end{array}$ & $\begin{array}{l}\text { Total Calories } \\
\text { Consumed } \\
\text { Due to } \\
\text { Walking (kcal) }\end{array}$ & $\begin{array}{c}\text { Total Calories } \\
\text { Consumed Due to } \\
\text { Lifestyle Activities } \\
\text { (kcal) }\end{array}$ & $\begin{array}{c}\text { Number } \\
\text { of Daily } \\
\text { Steps } \\
\text { (Step) }\end{array}$ \\
\hline $\begin{array}{l}\text { Physical } \\
\text { breathing effort }\end{array}$ & 0.3 & 0.28 & 0.34 & 0.15 & 0.35 & 0.12 & 0.41 & 0.19 & 0.34 & 0.34 \\
\hline Air hunger & 0.31 & 0.2 & -0.12 & -0.11 & -0.17 & -0.12 & -0.17 & $-0.47 *$ & 0.37 & -0.11 \\
\hline Tightness & 0.41 & 0.15 & -0.05 & -0.16 & -0.07 & 0.13 & 0.34 & -0.12 & 0.18 & 0.89 \\
\hline $\begin{array}{l}\text { Mental } \\
\text { breathing effort }\end{array}$ & 0.19 & -0.02 & -0.21 & -0.13 & 0.07 & -0.07 & -0.26 & -0.28 & 0.1 & -0.15 \\
\hline Hyperpnea & 0.17 & 0.26 & 0.03 & 0.01 & -0.23 & -0.38 & -0.20 & -0.18 & 0.41 & -0.21 \\
\hline Unpleasantness & -0.14 & 0.14 & 0.21 & 0.33 & 0.12 & 0 & -0.13 & 0.09 & 0.06 & -0.01 \\
\hline Depressed & 0.19 & 0.15 & 0 & -0.15 & $-0.49 *$ & -0.26 & -0.03 & -0.05 & 0.33 & 0.03 \\
\hline Anxious & 0.24 & -0.11 & -0.22 & -0.14 & -0.32 & -0.07 & 0.1 & -0.44 & $-0.46 *$ & -0.26 \\
\hline Frustrated & 0.06 & -0.20 & -0.17 & -0.35 & -0.12 & 0.26 & $0.45 *$ & -0.09 & -0.03 & -0.29 \\
\hline Angry & 0.18 & -0.18 & -0.30 & -0.30 & -0.06 & 0.22 & 0.36 & -0.34 & 0.06 & -0.3 \\
\hline Afraid & 0.21 & -0.12 & -0.14 & -0.21 & -0.19 & 0.21 & 0.4 & -0.25 & 0.09 & -0.15 \\
\hline
\end{tabular}

Notes: Data represent Spearman correlation coefficients. *And the bold font denote p-value less than 0.05 .

Abbreviation: MET, metabolic Equivalent. 
and $\mathrm{FEV}_{1}(\%)$ suggests that it is an important finding in determining the therapeutic effect of bronchodilation.

The present study suggested that the time of physical activity intensity of 1 and 2 METs to the total was greater and intense physical activity was lower. This observation is consistent with studies showing that COPD patients have more sedentary time and less physical activity than asthmatic and bronchiectasis patients. $^{31}$ However, we observed no association between exertional multidimensional dyspnea characteristics and the duration of low-intensity activity. This could indicate that improvement in breathlessness was not a significant contributor to the improvement in lowintensity physical activity.

The Japanese version of the MDP was completed within a few minutes by most participants. The MDP and all its derivative works are copyrighted by the developers, and the original reference should be cited by all papers using MDP. ${ }^{2}$ The original MDP and all its translations were distributed by the Mapi Research Trust (https://epro vide.mapi-trust.org), which should be contacted for any inquiry about the questionnaire and the requirements regarding its use.

The limiting factor in this study was the small sample size of the population, which only allowed us to examine univariate relationships. In addition, the study included female patients with COPD, and there may be gender effects on physical activity and multidimensional dyspnea properties. Therefore, future studies with larger numbers of patients are warranted.

\section{Conclusion}

In this study, we developed a linguistically validated Japanese translation of the MDP. The results suggest that air hunger is associated with avoidance of physical activity, while the emotional aspects of dyspnea affect both the amount and intensity of physical activity; therefore, a detailed assessment of multidimensional aspects of dyspnea is needed in clinical situations.

\section{Summary at Glance}

This study is the linguistic validation of the Japanese version of the multidimensional dyspnea profile and the first study to investigate the relationship between exertional multidimensional dyspnea and the profile of physical activity in patients with COPD.
Abbreviations

COPD, chronic obstructive pulmonary disease; $\mathrm{FEV}_{1}$, forced expiratory volume in $1 \mathrm{~s}$; FVC, forced vital capacity; MVV, maximal voluntary ventilation; MDP, multidimensional dyspnea profile; $\mathrm{P}_{\mathrm{I}}$, max, maximal inspiratory pressure; METs, metabolic equivalents.

\section{Ethics Approval and Informed Consent}

The study was approved by the institutional ethics review board of Himeji Dokkyo University (Himedookusei-1910) and was conducted in accordance with the Declaration of Helsinki. Informed consent was obtained in the form of opt-out by displaying a notice at the clinic.

\section{Acknowledgments}

We would like to thank Prof. Banzett for permission and help with the translation process.

\section{Funding}

This research was funded by Grants-in-Aid for Scientific Research from the Ministry of Education, Culture, Sports, Science and Technology (21K11206 and 18K17741, 19H03984). Re-search Grants for Osaka Gas Group Welfare Foundation (H28 and H29), Research Grant for Furukawa Medical \& Welfare Foundation (H31 and R1).

\section{Disclosure}

The authors report no conflicts of interest in this work.

\section{References}

1. Parshall MB, Carle AC, Ice U, Taylor R, Powers J. Validation of a three-factor measurement model of dyspnea in hospitalized adults with heart failure. Heart Lung. 2012;41(1):44-56. doi:10.1016/j. hrtlng.2011.05.003

2. Banzett RB, Pedersen SH, Schwartzstein RM, Lansing RW. The affective dimension of laboratory dyspnea: air hunger is more unpleasant than work/effort. Am J Respir Crit Care Med. 2008;177 (12):1384-1390. doi:10.1164/rccm.200711-1675OC

3. Waschki B, Kirsten A, Holz O, et al. Physical activity is the strongest predictor of all-cause mortality in patients with COPD: a prospective cohort study. Chest. 2011;140(2):331-342. doi:10.1378/chest.10-2521

4. Garcia-Aymerich J, Lange P, Benet M, Schnohr P, Anto JM. Regular physical activity reduces hospital admission and mortality in chronic obstructive pulmonary disease: a population based cohort study. Thorax. 2006;61(9):772-778. doi:10.1136/thx.2006.060145

5. Garcia-Aymerich J, Farrero E, Felez MA, et al. Risk factors of readmission to hospital for a COPD exacerbation: a prospective study. Thorax. 2003;58(2):100-105. doi:10.1136/thorax.58.2.100

6. Garcia-Rio F, Rojo B, Casitas R, et al. Prognostic value of the objective measurement of daily physical activity in patients with COPD. Chest. 2012;142(2):338-346. doi:10.1378/chest.11-2014 
7. Garcia-Rio F, Lores V, Mediano O, et al. Daily physical activity in patients with chronic obstructive pulmonary disease is mainly associated with dynamic hyperinflation. Am J Respir Crit Care Med. 2009;180(6):506-512. doi:10.1164/rccm.200812-1873OC

8. Burge AT, Cox NS, Abramson MJ, Holland AE. Interventions for promoting physical activity in people with chronic obstructive pulmonary disease (COPD). Cochrane Database Syst Rev. 2020;4: CD012626. doi:10.1002/14651858.CD012626.pub2

9. Kanezaki M, Terada K, Tanabe N, Shima H, Hamakawa Y, Sato S. Effects of sarcopenia on ventilatory behavior and the multidimensional nature of dyspnea in patients with chronic obstructive pulmonary disease. J Am Med Dir Assoc. 2021;22(4):827-833.

10. Kanezaki M, Terada K, Tanabe N, Shima H, Hamakawa Y, Sato S. Exertional multidimensional dyspnoea predicts exacerbation in stable outpatients with COPD. ERJ Open Res. 2021;7:00150-02021.

11. Faisal A, Alghamdi BJ, Ciavaglia CE, et al. Common mechanisms of dyspnea in chronic interstitial and obstructive lung disorders. $\mathrm{Am}$ $J$ Respir Crit Care Med. 2016;193(3):299-309. doi:10.1164/ recm.201504-08410C

12. Manning HL, Shea SA, Schwartzstein RM, Lansing RW, Brown R, Banzett RB. Reduced tidal volume increases "air hunger" at fixed PCO2 in ventilated quadriplegics. Respir Physiol. 1992;90(1):19-30. doi:10.1016/0034-5687(92)90131-F

13. Binks AP, Evans KC, Reed JD, Moosavi SH, Banzett RB. The time-course of cortico-limbic neural responses to air hunger. Respir Physiol Neurobiol. 2014;204:78-85. doi:10.1016/j.resp.2014.09.005

14. Banzett RB, O'Donnell CR, Guilfoyle TE, et al. Multidimensional Dyspnea Profile: an instrument for clinical and laboratory research. Eur Respir J. 2015;45(6):1681-1691. doi:10.1183/09031936.00038914

15. Williams M, Cafarella P, Olds T, Petkov J, Frith P. The language of breathlessness differentiates between patients with COPD and age-matched adults. Chest. 2008;134(3):489-496. doi:10.1378/chest.072916

16. Bech CS, Noerholm V, Bove DG, Poulsen I. Danish translation and linguistic validation of the multidimensional dyspnea profile. Eur Clin Respir J. 2021;8(1):1905498. doi:10.1080/20018525.2021.1905498

17. Ekstrom M, Sundh J. Swedish translation and linguistic validation of the multidimensional dyspnoea profile. Eur Clin Respir J. 2016;3:32665. doi:10.3402/ecrj.v3.32665

18. Stump A, Schroder M, Domanski U, Franke KJ, Nilius G. [German translation and linguistic validation of the Multidimensional Dyspnea Profile (MDP)]. Pneumologie. 2019;73(8):470-473. German. doi:10.1055/a-0861-1516

19. Belo LF, Rodrigues A, Vicentin AP, et al. A breath of fresh air: validity and reliability of a Portuguese version of the Multidimensional Dyspnea Profile for patients with COPD. PLoS One. 2019;14(4):e0215544. doi:10.1371/journal.pone.0215544
20. Wild D, Eremenco S, Mear I, et al. Multinational trials-recommendations on the translations required, approaches to using the same language in different countries, and the approaches to support pooling the data: the ISPOR patient-reported outcomes translation and linguistic validation good research practices task force report. Value Health. 2009;12 (4):430-440. doi:10.1111/j.1524-4733.2008.00471.x

21. Wild D, Grove A, Martin M, et al. Principles of good practice for the translation and cultural adaptation process for patient-reported outcomes (PRO) Measures: report of the ISPOR task force for translation and cultural adaptation. Value Health. 2005;8(2):94-104. doi:10.1111/j.1524-4733.2005.04054.x

22. The Japanese Respiratory Society. JRS COPD guideline Edition 5 updated current concept for clinical management of COPD in Japan; 2018.

23. Kanezaki M, Terada K, Ebihara S. Effect of olfactory stimulation by L-menthol on laboratory-induced dyspnea in chronic obstructive pulmonary disease. Chest. 2020;157:1455-1465. doi:10.1016/j. chest.2019.12.028

24. Borel B, Wilkinson-Maitland CA, Hamilton A, et al. Three-minute constant rate step test for detecting exertional dyspnea relief after bronchodilation in COPD. Int $J$ Chron Obstruct Pulmon Dis. 2016;11:2991-3000. doi:10.2147/COPD.S113113

25. Hirano T, Doi K, Matsunaga K, et al. A novel role of growth differentiation factor (GDF)-15 in overlap with sedentary lifestyle and cognitive risk in COPD. J Clin Med. 2020;9(9):2737. doi: $10.3390 /$ jcm 9092737

26. Oshima Y, Kawaguchi K, Tanaka S, et al. Classifying household and locomotive activities using a triaxial accelerometer. Gait Posture. 2010;31(3):370-374. doi:10.1016/j.gaitpost.2010.01.005

27. Nagayoshi S, Oshima Y, Ando T, et al. Validity of estimating physical activity intensity using a triaxial accelerometer in healthy adults and older adults. BMJ Open Sport Exerc Med. 2019;5(1):e000592. doi:10.1136/bmjsem-2019-000592

28. Kanda M, Minakata Y, Matsunaga K, et al. Validation of the triaxial accelerometer for the evaluation of physical activity in Japanese patients with COPD. Intern Med. 2012;51(4):369-375. doi:10.2169/ internalMedicine.51.6441

29. Alexander-Miller S, Davenport PW. Perception of multiple-breath inspiratory resistive loads in males and females. Biol Psychol. 2010;84(1):147-149. doi:10.1016/j.biopsycho.2010.03.004

30. Lewthwaite H, Li PZ, O'Donnell DE, Jensen D. Multidimensional breathlessness response to exercise: impact of COPD and healthy ageing. Respir Physiol Neurobiol. 2021;287:103619. doi:10.1016/j. resp.2021.103619

31. Cordova-Rivera L, Gardiner PA, Gibson PG, Winkler EAH, Urroz PD, McDonald VM. Sedentary time in people with obstructive airway diseases. Respir Med. 2021;181:106367. doi:10.1016/j. rmed.2021.106367

\section{Publish your work in this journal}

The International Journal of COPD is an international, peer-reviewed journal of therapeutics and pharmacology focusing on concise rapid reporting of clinical studies and reviews in COPD. Special focus is given to the pathophysiological processes underlying the disease, intervention programs, patient focused education, and self management protocols. This journal is indexed on PubMed Central, MedLine and CAS. The manuscript management system is completely online and includes a very quick and fair peer-review system, which is all easy to use. Visit http://www.dovepress.com/testimonials.php to read real quotes from published authors. 